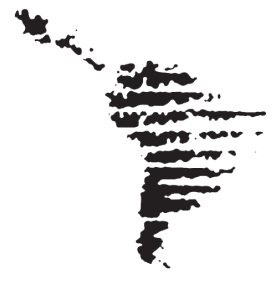

\title{
Geroidentidad social: Una reflexión sobre las definiciones, adjudicaciones, leyes, programas sociales y garantías humanas de las personas de la tercera edad en México
}

\section{Social geroidentity: a reflection about definitions, allotments, laws, social programs and human guarantees for the people of the third age from México}

\author{
Jesús Alberto Palma Hernández ${ }^{1}$
}

\section{Resumen}

El presente ensayo tiene como objetivo principal enunciar las diversas formas en las que se concibe a las personas de la tercera edad, desde la perspectiva de quien se encuentra fuera de dicho segmento e, igualmente, desde el punto de vista de quien es una persona adulta mayor. También se hace referencia a algunas leyes, programas sociales y garantías humanas que se crean para la protección y beneficio de dichas personas en México, con la finalidad de identificar indicadores que puedan dar cuenta sobre las actividades que a nivel social les aquejan. En conjunto con lo anterior y a partir de la teoría de las identidades sociales que propone Gilberto Giménez y que se usa como sustento de esta reflexión, se obtienen tres categorías de análisis (atributos, pertenencia y narrativa biográfica) que permiten el origen de una propuesta titulada "Geroidentidad social".

Palabras clave: Tercera edad, leyes, identidad social, derechos humanos, programas sociales

\begin{abstract}
The following paper has the objective of enouncing the many ways third age people are conceived from the perspective of the ones that are out of this segment, including the others that belongs to it. In the same way, this paper refers to some laws, social programs and human guarantees that were created to benefit and protect that people in Mexico, and which have the purpose of identifying some indicators that might tell about those social activities related to them. Based on those elements and the Theory of Social Identities from Gilberto Gimenez, the main argument of this reflection, three analysis categories are foud (attributes, membership and biographical narrative) that allows to create a proposal that is called "Social Geroidentity".
\end{abstract}

Keywords: third age, laws, social identity, human rights, social programs.

1 Mexicano. Licenciado en Comunicación por la Universidad de Ixtlahuaca CUI en el Estado de México, México. Actualmente es auxiliar digitalizador para la empresa farmoquímica Signa S.A. de C.V. dentro de su Centro de Documentación e Información. Participó en el XVI Certamen de Ensayo sobre Derechos Humanos que promueve la Comisión de Derechos Humanos del Estado de México, en el que obtiene el segundo lugar con el ensayo titulado: "La trata de personas como una práctica social cotidiana desde la perspectiva tecnológica-digital".

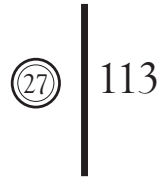




\section{Sobre la tercera edad y otros conceptos}

Los hombres y mujeres han sido partícipes de la clasificación, denominación y reglamentación de las formas que conducen al ser humano dentro de la sociedad, así mismo, las pautas por las cuales se pueden reconocer cosas, objetos y a sus semejantes mediante los significados que han sido instaurados y que se actualizan constantemente. Si bien existen diversas maneras de diferenciar al sujeto social mediante aspectos geográficos, demográficos, psicológicos, físicos y biológicos, estos mismos pueden proveer un sistema de agrupación de acuerdo con sus características $\mathrm{y}$ formar un segmento poblacional.

La lógica de la clasificación gira en torno a las características que visible o perceptiblemente se encuentran explícitas en un sujeto, por ejemplo, todo aquello que por el ojo humano puede ser visto y que, a su vez, es resguardado en la mente y memoria para así crear un patrón de reconocimiento. Así como en la era en donde se definía que el hombre era el proveedor de alimento y sustento al hogar a partir del trabajo, y la mujer era aquella que mantenía las relaciones administrativas y afectivas dentro de lo que se denomina familia ${ }^{2}$.

Ahora bien, dentro del seno familiar nuclear se encuentra, igualmente, una clasificación de roles, en los cuales se

2 La clasificación de las actividades o del trabajo dentro del círculo familiar lo ejemplifica Larissa A. de Lomnitz (1998) cuando menciona los albergan las referencias para denominar al hombre como padre, a la mujer como madre y al producto de su relación como los hijos, ya sean del sexo masculino o femenino. De modo cronológico, a nivel biológico, se habla de dos generaciones, la primera, que es la condición de ser un hijo, en su caso, niño o niña o joven, posteriormente, una segunda generación, la de los padres y madres que son adultos. Entonces, una tercera generación tendría que ser la de las personas antecesoras de los padres, madres, abuelas y abuelos de los hijos, que guardan una condición de filiación, que jurídicamente se entiende como aquel hecho natural relacionado con el ámbito biológico de la generación (Galindo, 1981).

Luego, entonces, con el acercamiento previo se puede comenzar a introducir la definición de "tercera edad", de donde se puede entender que es una última fase de la vida biológica de los hombres y mujeres, pues no hay una siguiente etapa y está referida a un parámetro de tiempo indefinido ${ }^{3}$, e incluso su inicio sufre de cambios constantes debido a la estimación de esperanza de vida que se especula es

roles desempeñados en Cerrada del Cóndor, una zona de barrios o barriada en la parte suroccidental de la Ciudad de México, en la que describe que la mujer es considerada como servil, una sustituta de madre y representante o pilar de la familia. Por otro lado, el hombre es dibujado como fuente de trabajo, pero también, desde la perspectiva femenina, como alguien emocionalmente inmaduro, es decir, un niño grande al resguardo de la mujer o esposa.

3 En diciembre de 2014, la Secretaria de Desarrollo Social (SEDESOL) en México considera que la edad pertinente para la obtención del Programa 
la idónea, aunque, no por eso se pierden las características ni garantías y responsabilidades como ciudadanía.

Primeramente, existe un constante devenir de definiciones y conceptos que parecieran ser sinonímicos, por ejemplo, cuando se menciona la tercera edad, se especula que sea un lapso, es decir, se puede medir en años, para el caso de la persona adulta mayor, se entiende que es un sujeto social y puede ser descrito mediante un ejercicio de observación, aunque, también puede ser referenciado (por instituciones) de acuerdo con la cantidad de años.

Finalmente, la vejez sería una condición psicosocial y no únicamente una edad biológica, sino que adquiere características referidas al adentramiento de la mente y a la labor del sujeto dentro de su entorno y sus diversas esferas: lo político, religioso, cultural, cívico, etc. A continuación, se realiza un ejercicio reflexivo sobre las diferencias y similitudes entre los conceptos previos y la conexión que guardan.

María Rosario Limón (1992, p.167) considera que la tercera edad:

... es una etapa, la más avanzada, de la evolución y desarrollo personal, que por sus características especiales

Pensión para Adultos Mayores sean los 65 años, esto debido al incremento de este segmento de la población y que en una proyección al 2050 (CONAPO), existirá una tendencia de proporción a que haya 73 adultos mayores por cada 100 menores de 15 años (DOF, 2014). merece ser tratada con mayor atención y cuidado, pero que para muchos de sus componentes, se convierte con frecuencia en una etapa de marginación y abandono.

La cita anterior da cuenta de dos aspectos, el primero, la tercera edad sí está vinculada a un tiempo dentro del desarrollo de los sujetos adjudicado al indicador de la edad; el segundo, la tercera edad se refiere a aquel sujeto que se encuentra vulnerable ante ciertas situaciones, ya sea por sus capacidades físicas, emocionales o de salud que decrecen, y que como consecuencia sufre violencia de tipo cultural ${ }^{4}$, pues carece de evidencia perceptible como una lesión, se encuentra en el rango de lo invisible y tiene repercusiones en la integridad del mismo sujeto desde diversas perspectivas, como la exclusión social que trae consigo el desentendimiento de la familia y la pérdida de los valores de esta.

Por otro lado, también surge el término adulto mayor o personas adultas mayores, que es introducido por la Ley de los Derechos de las Personas Adultas Mayores (LDPAM), en dónde en su artículo $3^{\circ}$, fracción I, se plantea que son: "aquellas que cuenten con sesenta años o más de edad y que se encuentren domiciliadas o en tránsito en el territorio

4 La violencia cultural son los aspectos de la cultura desde el rubro simbólico, que pueden ser utilizados para justificar o legitimar la violencia directa y estructural (Galtung, 2003); para el 
nacional" (DOF, 2015, p. 2). Se entiende que el marco jurídico se rige, de igual manera, por un estándar de edad que a veces tiende a ser un tanto variable, dependiendo de cada institución que defina al sujeto de estudio, pero que resulta siempre concentrado en ese periodo de la edad biológica de los 60 años en adelante y que cuenta con sus derechos de ciudadanía.

caso de la persona de la tercera edad, sufrir violencia directa es el maltrato físico que deviene de la incapacidad social de algunos sujetos que no comprenden el cambio biológico, físico y psicológico de estas personas, quienes, además de no estar satisfechos con las lesiones, buscan excluir y marginar a este segmento poblacional para asumir un rol de supremacía (violencia estructural), lo mismo puede suceder de manera inversa y como resultante emerge la sumatoria de tres tipos de violencia para un solo caso.
Aunque pareciera que no hay otra forma de describir a las personas de la tercera edad o a las personas adultas mayores, existen particularidades visibles que las hacen diferenciarse de la demás población y que han formado en la mente y memoria de la ciudadanía en general un imaginario social ${ }^{4}$ que permite legitimar dichas características y perpetuarlas, mencionarlas incurriría en un acto paradójico debido a la experiencia personal con la que se cuenta y contradecirlo sería negar la realidad que se vive. Un sitio en línea hace alusión a dichas características y otros aspectos que se presentan en la siguiente tabla:

\section{Tabla 1}

Particularidades de una persona de la tercera edad desde el enfoque de un tercero

\section{Características de la tercera edad}

1. Empiezan a padecer enfermedades biológicas como: artrosis, diabetes, cataratas, Párkinson, osteoporosis, y enfermedades cardiovasculares, etc.

2. Dentro de las enfermedades neurológicas o mentales están: Alzhéimer, demencia senil.

\section{Enfermedades sociales}

3. Aislamiento social.

4. Escasa o nula oportunidad de trabajo.

5. Poco o nulo acceso a parques donde puedan encontrar juegos apropiados a su edad para la realización de actividades físicas.

6. Discriminación familiar, si no aporta económicamente con su pensión de jubilación.

7. Su diferencia cultural hace que su autoestima esté muy baja, si no tiene independencia económica, necesariamente debe retribuir con trabajo físico su estadía en el hogar.

Nota: Elaboración propia con información del blog titulado "La tercera edad. Características del adulto mayor" (Hora Buena. Para que todas tus horas sean buenas, 2011).

$4 \quad$ Es la percepción del mundo desde la psique del sujeto, de lo que es real o lo que se cree es real (Castoriadis, 1997). 
La relevancia dela tabla anterior reside primordialmente en la subjetividad y cambiante opinión pública ${ }^{5}$, que ahora también puede estar inmersa en el mundo de las tecnologías y sus diversas plataformas, como sitios web, blogs, salas de chat, redes sociales, etc., y que permiten bosquejar el panorama actual sobre el cómo ve la sociedad al segmento poblacional de la tercera edad o personas adultas mayores, esto como un primer acercamiento y clasificación de roles sociales, además de mantener latente su imaginario social.

Pero, ¿por qué relacionar a las personas inmersas en la tercera edad con cuadros patológicos?, ¿a qué se debe que también existan males sociales vinculados a estos grupos? Es un patrón que constantemente se perpetúa mediante el principio de la clasificación, de aquella acción de catalogar y determinar las actividades para cada uno de los individuos que integran la sociedad a partir de lo que son, saben hacer, suscaracterísticas y sus contrapartes, por ejemplo, los defectos, carencias, discapacidades, etc. Muchas veces, es la creación de un estigma que es heredado a nivel generacional, que se alimenta de experiencias, medios de comunicación, ideologías, posturas

$5 \quad$ La opinión pública a veces se pudiera entender como un ejercicio subjetivo, como un aspecto personal e interno; pero, cuando se comparte dicha opinión y existe cierta concordancia con otras, se transforma en una cuestión de carácter plural que legitima acciones, significados o autoridad política y su mismo orden (Heller, 2000). negativas; la falta de valores morales y empatía es la verdadera enfermedad social preocupante.

Un último concepto aunado también a la tercera edad es la vejez que, como sus otros sinónimos, alterna algunas características y se define como: “... la última etapa del ciclo de la vida donde se aprecia el resultado de todas las experiencias, transformaciones y aprendizajes vividos en las etapas anteriores, y donde se acepta la trayectoria de vida con satisfacción" (Rodríguez, 2011, p.13).

Es crucial acentuar que se aprecian más que referentes biológicos y médicos sobre lo que es la vejez, una persona de la tercera edad o adulta mayor, que existe un tipo de indicio histórico cuando se hace alusión a la experiencia, y que guarda consigo cierta "sabiduría" que se transmite mediante el nexo familiar o profesional.

Nobastaconlasdesignacionesmédicas, biológicas y físicas que describen a las personas que se encuentran dentro de la tercera edad, puesto que las enfermedades no eligen siempre a dicha población y tienden a incubarse en otros cuerpos, tal vez más sanos, tal vez más jóvenes. Tampoco habría que designarles un grado jerárquico a los significados de los conceptos, pues todos se conectan con el mismo sujeto. ¿Qué hay de las actividades sociales?, ¿por qué es marginada y eclipsada la persona adulta mayor si sigue siendo ciudadana?, ¿ya no tiene

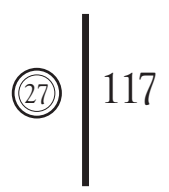


participación dentro de los ámbitos de la cotidianidad que nos rodean?, ¿es la supuesta mayoría joven o adulta la que decide qué se debe hacer? La posible respuesta se resume en dos palabras: grupos vulnerables.

\section{¿Qué son los grupos vulnerables?}

De acuerdo con el INEGI ${ }^{6}$ y hasta el año 2010, la República Mexicana contaba con 112.336 .538 habitantes, de los cuales, un $9.1 \%$ eran personas de 60 años y más, lo que quiere decir que, al menos 10.222.625 ciudadanos, entre hombres y mujeres, concentraban a la población de la tercera edad; al respecto el Estado de México es una de las entidades federativas con mayor número de habitantes de dicho segmento, pues del total estatal que fue de 15.175 .862 habitantes, un $7.6 \% \quad(1.153 .365,51$ personas) fueron adultos mayores.

Es decir, una cantidad considerable de la población mexicana pertenece a los grupos vulnerables; pero, ¿qué son estos grupos?, ¿por qué son vulnerables? Las cifras parecen siempre ser el foco de atención del público lector, ciudadanía, televidentes e instituciones, pero nunca se toma en cuenta el trasfondo social que esto encierra, no se analiza con detenimiento que existen formas de erradicar males concernientes a estas agrupaciones mediante acciones

6 Para su consulta general, visite el sitio web del Instituto Nacional de Estadística y Geografía (INEGI) desde su página principal: http:// www3.inegi.org.mx/sistemas/mexicosifras/ default.aspx sencillas, como el respeto, tolerancia e, incluso, el reconocimiento. Estos tendrían que ser valores morales ${ }^{7}$ previamente instruidos que se fortalecen mediante la práctica diaria; la finalidad de estos es mostrar que todas las personas estamos expuestas a los mismos males sociales e, igualmente, somos diversas.

Sin embargo, se asume una postura de no responsabilidad con personas ajenas a nuestro entorno; hasta cierto punto, existe un grado de exclusión que aleja a quienes integran los antes mencionados grupos y permite que se les conciba como vulnerables, por no ser una mayoría, por no desempeñar las mismas actividades socionormadas de la cotidianidad, por contar con prácticas culturales diferentes, etc., y que esta situación desemboque en lo vulnerable, entendido por la Real Academia de la Lengua Española (2016) como "todo aquello que puede ser herido o recibir lesión, física o moralmente", desde luego que, todo el mundo puede tener dicha característica en medidas diferentes.

Luego, entonces, los grupos vulnerables son aquellos segmentos sociales susceptibles a cambios y que, por consiguiente, tienen repercusiones

7 Los valores en su sentido amplio son aquellas cualidades reales de las personas, las acciones, los sistemas o las cosas que son distinguibles y que se adecuan de acuerdo con cada cultura e historia. Los valores morales giran en torno a situaciones que guardan una relación antagónica o una antonimia, por ejemplo: justicia/injusticia. Se vislumbra sobre todas aquellas acciones que resultan ser positivas ante otras que son negativas y viceversa (García, 2001). 
graves como la agresión física, verbal, psicológica, moral u otra que no permite su desarrollo pleno y que, de alguna manera, se encuentran eclipsados por una mayoría relativa, que desempeña actividades que estos no pueden realizar por diversos factores, ya sean naturales, sociales, culturales, políticos, religiosos, etc.

Mencionan Valadés y Gutiérrez (2001) que un grupo vulnerable es:

...aquel que, en virtud de su género, raza, condición económica, social, laboral, cultural, étnica, lingüística, cronológica y funcional sufren la omisión, precariedad o discriminación en la regulación de su situación por parte del legislador federal o local del orden jurídico nacional. (p. 227)

Entre tanto, se considera que esos grupos forman parte de una minoría cuasi-invisible que adquiere relevancia y una presencia mucho más amplia cuando se habla de ella en conjunto, pues, por sus diferentes características, tiende a ser separada, pero la une el mismo sistema social. A continuación, se hace mención de las segmentaciones consideradas como grupos vulnerables (Valadés y Gutiérrez, 2001, p. 227):

a) La mujer pobre jefe de hogar, con niños a su cargo, y responsable del sostenimiento familiar.

b) Menores y adolescentes en situación de riesgo social (niños en riesgo de salir del hogar, menores infractores y menores víctimas de violencia física, sexual o psicológica en el seno familiar, menores con padecimientos adictivos).

c) Los menores que viven en la calle o los menores que, no obstante tener un hogar, a causa de la desintegración familiar o problemas de otra índole pasan todo el día en la calle.

d) Los menores trabajadores (pepena, estiva, mendicidad, venta ambulante, limpia de parabrisas y actuación en la vía pública).

e) Las personas de la tercera edad.

f) Las personas discapacitadas.

g) La población rural e indígena que se encuentra afectada en forma alarmante por la pobreza.

h) Las mujeres pobres, embarazadas y en estado de lactancia.

i) Los jóvenes y las mujeres pobres afectadas por el desempleo.

j) Los trabajadores pobres del sector informal.

k) Los excluidos de la seguridad social.

1) Las mujeres que sufren discriminación política social.

m) Los pueblos indígenas.

La lista podría seguir aumentando de acuerdo con el enfoque, tanto 
disciplinario como cultural que se busque aplicar para este caso. Ahora bien, la mayoría de los ejemplos anteriores se refiere a una diferencia o discrepancia, ya sea económica, étnica, física, de edad y otras; aunque, en muchas de las ocasiones existe una sumatoria de diferentes carencias para una sola persona, esto no quiere decir que sean más vulnerable, sino que se lidia con segmentos mucho más diversos (o extensos), por ejemplo, un indígena de la tercera edad o una mujer pobre excluida socialmente y, además, de la tercera edad.

Es un hecho que la discriminación es uno de los indicadores por los cuales se puede definir a las personas de la tercera edad como un grupo vulnerable, no simplemente por una cuestión referida a las minorías, ni a la falta de capacidades o habilidades debidas a la edad, sino que también es un problema ideológico encasillado en un imaginario que, como se mencionó con anterioridad, se perpetúa.

En la tabla 2, elaborada a partir de información proporcionada por el Consejo Nacional para Prevenir la Discriminación (CONAPRED, 2011), en su Encuesta Nacional sobre Discriminación en México, se enuncian algunos de los principales problemas que consideran las personas adultas mayores de alta injerencia en México para esta misma comunidad mediante el cuestionamiento: Hoy, ¿cuál cree que es el principal problema para la gente de su edad en México?

Tabla 2

Principales problemas de las personas adultas mayores desde una perspectiva individual

\begin{tabular}{clc}
\hline \multicolumn{1}{c}{ Problema } & Porcentaje \\
\hline 1. & Dificultad para encontrar trabajo & $36 \%$ \\
2. & Salud & $13.9 \%$ \\
3. & Discriminación, intolerancia & $9.2 \%$ \\
4. & NS/NC (No sabe, no contesta) & $7.8 \%$ \\
5. & Falta de apoyo y oportunidades del gobierno & $7.3 \%$ \\
6. & Económico & $6.9 \%$ \\
7. & Otros & $5.3 \%$ \\
8. & Soledad, abandono & $4.1 \%$ \\
9. & Inseguridad & $2.6 \%$ \\
10. & No ser autosuficiente & $1.5 \%$ \\
11. & Problemas de transporte y de salir a la calle & $1.5 \%$ \\
12. & Discapacidad & $1.2 \%$ \\
13. & Falta de cuidado de los familiares & $1.2 \%$ \\
14. & Maltrato & $0.7 \%$ \\
15. & Bajo nivel educativo & $0.6 \%$ \\
16. & Falta de espacios adecuados (asilos, recreativos) & $0.2 \%$ \\
\hline
\end{tabular}

Nota: Elaboración propia con información de la Encuesta Nacional sobre Discriminación en México (Enadis-2010, CONAPRED, 2011). 
Si bien la mayoría de los problemas anteriores pueden ser definidos como violencia en sus formas culturales $y$ estructurales, parten del fundamento de la discriminación ${ }^{8}$ y reiteran la constante vinculación de las personas de la tercera edad con una patología social de exclusión y rechazo por la falta de habilidades, diversidad ideológica, diferencias económicas y otras que amenazan la integridad física y moral de esta parte de la población, de ahí que se afirme que las personas adultas mayores son un grupo vulnerable, por la sensibilidad con la que cuentan desde las diversas esferas sociales que les rodean.

Cabe destacar que la familia también es un rubro de injerencia importante en el desarrollo de la persona adulta mayor, pues además de proveer resguardo, es el armazón por el cual comienzan a educarse las generaciones futuras o póstumas, es decir, sin la implementación de valores en el hogar, se seguirán perpetuando los modelos de actitud y comportamiento negativo o precario ante aquellas personas de la tercera edad que también forman parte del núcleo o círculo familiar, por eso se mencionó en el apartado anterior la relevancia de los roles familiares, de su división como

8 En Toluca, Estado de México, las personas adultas mayores consideran que la discriminación e intolerancia son los principales problemas que atañen a su comunidad y segmentación, pues al menos un $24.3 \%$, el porcentaje más alto a nivel zonas metropolitanas, se concentra en dicha ciudad, de acuerdo con la Encuesta Nacional sobre Discriminación en México /Enadis-2010 (CONAPRED, 2011). generaciones, porque son, tal vez, una medida de mejoramiento de las sociedades futuras, en donde se debe reparar o potenciar el funcionamiento de la primera institución social ${ }^{9}$ que conoce el hombre y la mujer.

Desde la perspectiva de las personas de la tercera edad, existe un aspecto por el cual se considera importante este segmento de la población dentro del rubro familiar a partir su clasificación como abuelo o abuela, pues fungen como quienes educan, proteguen o tutorean a sus nietos o nietas; son las figuras paternas más cercanas debido a cuestiones de ausentismo, provocado por diferentes causas como desempleo, edad (jóvenes/ madurez), falta de recursos económicos, fallecimiento, etc.

La mayoría de los abuelos asume la responsabilidad de hacer de padres de sus nietos en un periodo de sus vidas que está típicamente reservado para el retiro, dejando atrás sus sueños de jugar y de visitar por recreación. Estos abuelos precisan apoyo y acceso a recursos comunitarios que los ayuden a sobrellevar las cuestiones de paternidad

9 En algunas de las ocasiones se discrepa que la familia sea una institución social, pues más se le adjudica un derecho natural de filiación que se refiere a la pertenencia biológica, debido a la procreación; sin embargo, otros mencionan que no se les puede denominar a las familias como instituciones naturales, pues es una instancia de carácter humano, racional y cultural que sobrepasa lo natural (Burgos, 2005).

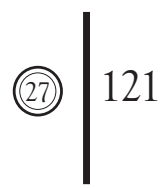


que surgen de su particular situación. También necesitan cuidar su propia salud y bienestar, para poder cumplir los desafíos y las demandas de los padres. (New York-Presbyterian Hospital, 2005, p.1)

Las formas de contrarrestar los efectos negativos en las personas de la tercera edad son los programas de apoyo y leyes o normas jurídicas, que además de ser un complemento de las garantías humanas con las que cuenta toda la ciudadanía a partir de la Constitución Política de los Estados Unidos Mexicanos y la Declaración Universal de los Derechos Humanos, son un aditivo que busca protegerles ante los males sociales que les aquejan; así también, tendrían que ser un soporte para poder solventar las necesidades emergentes en caso de sustituir a los padres o madres dentro de algunas familias.

Esos programas y leyes que colaboran de manera alternativa pueden ser una medida para hacer valer las garantías humanas de dichas personas; pues, al ser constantemente marginadas y eclipsadas con la cotidianidad invasiva (tecnológica) y cierta supremacía juvenil, se busca aminorar la condición de vulnerabilidad que se les adjudica y con la que viven día tras día. No por ello se debe asumir que existe un modelo universal para envejecer, o que la totalidad se encuentre incapacitada para continuar realizando labores comunes como trabajar o ser el sustento de una familia; sin embargo, son acciones que se realizan como un plan provisorio o a futuro:

Tarde o temprano, si primero no sobreviene la muerte, la vejez impone fatalmente el retiro del trabajo, convirtiendo a los adultos mayores en personas totalmente dependientes de los sistemas de transferencia. Cada sociedad dispone de mecanismos de redistribución de recursos que ponen de manifiesto los escenarios de vida a los que se enfrentan los adultos mayores. Ellos pueden subsistir de contribuciones suministradas por el Estado, de recursos proveniente de sus hogares y redes sociales y familiares de apoyo, de sus ahorros e inversiones acumuladas o bien de la caridad pública. (CONAPO, 1999, p. 20)

Dado que es incierto el contexto y las situaciones de las personas adultas mayores en sus diversas esferas o niveles, y se bosqueja muy mínimamente su realidad a nivel personal e individual, se promueven programas de apoyo junto con marcos jurídicos que puedan satisfacer algunas de las necesidades que más aquejan a este segmento de la población. En el siguiente apartado se hará una breve mención sobre algunos 
de los programas, leyes y normativas que benefician a las personas de la tercera edad y cómo pudieran estar relacionadas con las garantías y derechos humanos.

\section{Leyes y programas de apoyo para} las personas de la tercera edad en México como medida garante de los derechos humanos

Desde el momento en que al hombre y a la mujer se les reconoce la ciudadanía mexicana, se sabe que cuentan con los derechos y obligaciones que se encuentran concentrados en la Constitución Política de los Estados Unidos Mexicanos, así también, desde el momento en que nacen se benefician por la Declaración Universal de los Derechos Humanos hasta el fin de su vida.

Sin embargo, existen factores externos que no permiten que se satisfagan las necesidades necesarias de ciertos grupos por cuestiones culturales, ideológicas o económicas y, para ello, hay programasyleyes complementarias que buscan su desarrollo integral. En el caso de las personas de la tercera edad en México, se cuenta con la Ley de Los Derechos de las Personas Adultas (DOF, 2015) en donde se resaltan y demarcan, en el Capítulo II, los derechos de este segmento de la población, que se albergan en su artículo $5^{\circ}$ y de donde se desprenden nueve derechos fundamentales:
I De la integridad, dignidad y preferencia

II De la certeza jurídica

III De la salud, la alimentación y la familia

IV De la educación

V Del trabajo

VI De la asistencia social

VII De la participación

VIII De la denuncia popular

\section{Del acceso a los servicios}

Ahora bien, pareciera que muchos de estos derechos ya se encuentran en la Constitución Política de México; sin embargo, se hacen de carácter prioritario cuando una persona de la tercera edad los demanda, ya que, debido al constante arraigamiento del trabajo y los beneficios sociales en la ciudadanía joven y adulta (adultos jóvenes), pocas de las veces se hace caso de las necesidades de esta parte de la población. A partir de este documento, existen otros como la cartilla de los Derechos de los Adultos Mayores (CNDH, 2012), que desmiembra el marco jurídico anterior en otras 4 secciones que grosso modo se enuncian a continuación: 


\section{Tabla 3}

Cartilla de los derechos de los adultos mayores (división y clasificación)

\section{No discriminación y relaciones familiares}

1. No discriminar en razón de su edad.

2. Oportunidades de manera equitativa.

3. Apoyo institucional para garantizar sus derechos.

4. Protección ante los diversos tipos de violencia.

5. Protección familiar y social.

6. Mantener una relación familiar en caso deseparación, a no ser que la relación sea nociva, en ese caso no es aplicable.

7. Vivienda digna y decorosa.

8. Libertad de expresión.

\section{Frente a las autoridades}

9. Ser tratadas con dignidad y respeto cuando sean detenidas o sean víctimas de un delito/infracción.

10. Contar con asesoría jurídica gratuita y oportuna.

11. Realizar con libertad su testamento sin la intervención de una tercera persona.

\section{Protección de su salud}

12. Recibir información sobre las instituciones que pueden brindarle un servicio de salud integral.

13. Recibir atención médica en cualquiera de las instituciones del sistema nacional y estatal de salud.

14. Recibir orientación y asesoría respecto a su salud integral, física y mental.

15. Recibir seguridad social.

16. Poder integrarse a los programas de asistencia social cuando se encuentren en situaciones de abandono.

\section{Educación y trabajo}

17. Contar con un trabajo mediante la obtención de oportunidades igualitarias para su acceso (de acuerdo con sus cualidades y capacidades).

18. Recibir un ingreso propio mediante el desempeño de un trabajo remunerado.

19. Recibir educación y capacitación en cualquiera de sus niveles.

20. Tener libertad de asociación y reunión.

21. Participar en actividades culturales, deportivas y recreativas.

Nota: Elaboración propia con información de la cartilla de los Derechos de los Adultos Mayores (CNDH, 2012). 
Algunas de estas garantías están reflejadas dentro de los programas sociales, tanto estatales como federales a favor de las personas de la tercera edad y tienden a representar algunas de las formas en las que se busca integrar al segmento de la población adulta mayor, con el afán de brindarle apoyo y visibilidad ante los diversos males que le aquejan. Se toman como ejemplo directo los programas sociales que se muestran en la tabla 4 .

Las instituciones gubernamentales $y$ no gubernamentales tienen un papel relevante en la protección y validación de los derechos humanos de las personas de la tercera edad, así también, la acción conjunta de los programas sociales y la ciudadanía que permite la integración de este grupo a los ámbitos que más les convengan, desde el empleo hasta los servicios de salud.

Dado que existen diversas formas de equilibrar los beneficios sociales contra los males como la discriminación, el alejamiento y la violencia (que, ocasionalmente, no son una decisión personal por parte de las personas adultas mayores) y que buscan salvaguardar de estos mismos, también se debe tener en cuenta que las formas de apoyarles tienen diferentes perspectivas que van desde lo jurídico, hasta lo social, y que cruzan el sendero humano y llegan hasta las aras de lo moral.

Ahora bien, la definición tanto como segmento social y como grupo vulnerable, en sumatoria con los marcos jurídicos, leyes y programas de apoyo, dan cuenta de un aspecto que no se ha profundizado del todo sobre las personas de la tercera edad, este aspecto se encuentra inmerso en el derecho a la cultura, que opera desde la Ley de los Derechos de las Personas Adultas Mayores (LDPAM) (DOF, 2015), en su artículo $5^{\circ}$, fracción VII, inciso d, que indica que dicha población tiene el derecho a participar en la vida cultural, deportiva y recreativa de su comunidad; sin embargo, no es la cultura aquel aspecto que se pasa por alto, sino más bien la identidad social de las personas de la tercera edad, lo que constituye la propuesta principal del presente trabajo.

\section{Geroidentidad: La construcción de la identidad social de las personas de la tercera edad}

De entre todos los derechos humanos que se encuentran expresados en las leyes y programas sociales que refieren a las personas de la tercera edad, la identidad es uno de los ámbitos (y también derecho) que no se menciona con frecuencia. Su proveniencia pareciera ser un aspecto personal e individual, una peculiaridad que hace que los seres sociales se diferencien entre sí y otros más, que segmenta y que pretende agrupar, o clasificar, las pautas por las cuales cada quien debe responder $\mathrm{y}$ a las cuales corresponde pertenecer.

La identidad tiene origen en la cultura, esta última no considerada como 


\section{Tabla 4}

Comparación entre programas sociales a favor de la población adulta mayor desde la perspectiva estatal y federal en México

\begin{tabular}{|c|c|c|c|}
\hline $\begin{array}{l}\text { Nombre del } \\
\text { programa }\end{array}$ & Jurisdicción & Descripción & Incentivo \\
\hline $\begin{array}{l}\text { Programa de } \\
\text { Desarrollo } \\
\text { Social Gente } \\
\text { Grande }\end{array}$ & $\begin{array}{l}\text { Estatal } \\
\text { (Estado de } \\
\text { México) }\end{array}$ & $\begin{array}{l}\text { Es un programa que busca } \\
\text { favorecer el acceso de ali- } \\
\text { mentos, productos de lim- } \\
\text { pieza y aseo personal. }\end{array}$ & 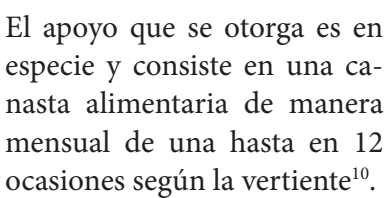 \\
\hline
\end{tabular}

$\begin{array}{ll}\begin{array}{l}\text { Programa de } \\ \text { Desarrollo } \\ \text { Social Gente } \\ \text { Grande }\end{array} & \begin{array}{l}\text { Estatal } \\ \text { (Estado de } \\ \text { México) }\end{array} \\ & \\ \text { Programa de } & \\ \begin{array}{l}\text { Desarrollo So- } \\ \text { cial Adultos en } \\ \text { Grande }\end{array} & \begin{array}{l}\text { Estatal } \\ \text { México) }\end{array}\end{array}$

Es un programa que tiene como propósito la integración a partir de los 60 años, a la vida productiva mediante su acceso a cursos de capacitación, que propician mejorar su economía.
El programa atiende a las personas mayores de 65 años en adelante y tiene cobertura a nivel nacional.
Se ofrecen cursos de capacitación, los insumos para estos mismos y material básico a cada persona beneficiaria.
Pensión para

Adultos Ma- Federal yores
Las personas beneficiarias reciben apoyos económicos de 589 pesos mensuales con entregas de 1,160 pesos cada dos meses ${ }^{11}$.
El programa es una plataforma para vincular una relación con los sujetos prestadores de servicios y empresas que desean incorporar a las personas de $60 \mathrm{y}$ más años de edad, promoviendo la inclusión laboral para este grupo etario.
Las empresas incorporan a personal adulto mayor al mercado laboral y bridan:

a) Sueldo base

b) Prestaciones de Ley

c) Contrataciones por hora, por jornadas, por proyecto o por servicios

d) En algunos casos prestaciones superiores a las de la Ley

10 Las vertientes para este programa son dos vías de acción, la primera se enfoca a la población adulta mayores de 60 a 69 años de edad y se otorgan apoyos que favorezcan el acceso de alimentos. La segunda vertiente está referida a mayores de 70 años de edad en adelante, y se otorgan apoyos que favorezcan el acceso de alimentos y a productos de aseo personal (GEM, 2014).

11 Además del apoyo económico que se otorga, se busca integrarles en grupos de crecimiento y jornadas informativas sobre temas de salud, se busca que obtengan facilidades de acceso a servicios y apoyos de instituciones como el INAPAM, así como también aquellas que ofrezcan actividades productivas y ocupacionales (SEDESOL, 2015).

Fuente: elaboración propia con base en datos del sitio en línea de la Secretaría de Desarrollo Social (SEDESOL, 2015), y la Gaceta del Gobierno del Estado de México con fecha del 30 de enero de 2014 (19) (GEM, 2014). 
aquello que está vinculado a la educación, tampoco al arte, sino como "una instancia simbólica de la producción y reproducción de la sociedad" (García-Canclini, 2004, p. 37), es decir, es el reflejo de la situación actual en la que se encuentran los sujetos y la ciudadanía, con todas sus desventuras y aciertos en sus diversas esferas que les rodean, de lo económico a lo religioso, de los sistemas de salud hasta las formas de gobierno, desde la marginación y discriminación hasta los derechos humanos, todo eso es la cultura, claro, una cultura contextualizada para un caso particular, las personas de la tercera edad, puesto que la cultura actúa de manera diferente para cada región, población o área geográfica.

Entonces, desde la perspectiva de Gilberto Giménez (2009, p. 27) la identidad "no es más que el lado subjetivo de la cultura considerada bajo el ángulo de su función distintiva", es decir, la particularización de la cultura mediante la interpretación y ejercicio hermenéutico del individuo como medida que distingue, y como ejercicio discriminante sin connotar una acción violenta, sino más bien de separación e incluso como referente de la diversidad social que aqueja el presente. La teoría que maneja Gilberto Giménez (2009) sobre las identidades sociales consta de tres categorías que permiten el análisis y construcción de esta a nivel social:

1. Atributos

2. Pertenencia

3. Narrativa biográfica
Así, también, se cuenta con un factor que permite legitimar la existencia de dicha identidad, como la estancia de los sujetos sociales dentro de los campos cognitivos: la memoria colectiva. A continuación, en la tabla 5 se ofrece un acercamiento a la identidad social de las personas de la tercera edad (geroidentidad social) mediante la información de los apartados anteriores y la teoría planteada por Giménez (2009).

Los atrib utos de las personas de la desembocan en los programas sociales mencionados en el apartado 3 , los que, por lo general, tienden a vincularlas con otros sitios de recreación y de capacitación en donde se tejen las redes de pertenencia (la interacción con sus semejantes), y que finalmente dan cuenta de cierta experiencia que se almacena y resguarda en la memoria de los mismos sujetos, conformándola como su propia narrativa biográfica, ya que el presente también se transforma en pasado.

Aunque, la memoria colectiva con respecto a la identidad se refiere al recuerdo personal y a la vez plural (Halbwachs, 2004), construido a partir de lo que se ve y lo que los demás individuos ven para poder legitimar ciertas instancias, la cual, para el caso de las personas de la tercera edad, resulta ser una paradoja, pues permite la perpetuación del estereotipo (o imaginario social) de ser un grupo vulnerable $y$, por tanto, 
Tabla 5

La teoría de la identidad social aplicada a las personas de la tercera edad

Identidad social (Giménez, 2009) / Categorías

Atributos

Son las características físicas, biológicas y perceptibles de los sujetos.
Pertenencia

Narrativa biográfica

Es la inclusión de la personalidad individual en una colectividad. Lugares y personas con quienes se mantiene una relación o vinculación.
Es la trayectoria personal del pasado que confiere sentido, así también el reconocimiento propio de los sujetos.

\section{Geroidentidad social (personas de la tercera edad)}

Atributos

${ }^{*}$ Características físicas:

- Cabello entre gris y blanco, piel arrugada, cuerpo encorvado.

${ }^{*}$ Son personas de 60 años en adelante.

${ }^{*}$ Son personas que sufren de enfermedades biológicas como:

- Artrosis, diabetes, cataratas, Párkinson, osteoporosis, y (enfermedades) cardiovasculares.

*Son un segmento poblacional que sufre de males sociales como la discriminación y marginación.
Pertenencia

Narrativa Biográfica
${ }^{*}$ Se le considera un grupo vulnerable.

${ }^{\star}$ Los sitios ${ }^{12}$ que regularmente frecuentan son:

-Parques, centros de capacitación, albergues, residencias diurnas y sitios de recreación entre los que se incluyen hoteles, museos, cines, balnearios, teatros, parques recreativos, zonas arqueológicas.

${ }^{\star}$ Los derechos humanos de las personas adultas mayores y las leyes o programas sociales vinculados a ellas tienden a unificar a este segmento de la población para después congregarlo dentro de espacios que hacen valer dichas garantías.
${ }^{\star}$ Este aspecto se refiere a la experiencia o etnografía de las personas adultas mayores $y$ personas de las tercera edad, es decir, a todo aquello que a nivel histórico ha realizado y que ha servido para la conformación de sociedades futuras.

12 Las redes de pertenencia fueron obtenidas de acuerdo con los beneficios que ofrece la afiliación al INAPAM (2016) mediante la obtención de su tarjeta.

Nota: Elaboración propia con fundamento en la obra de Gilberto Giménez (2009). 
un sujeto no activo. Cuando bien, como se mencionó con anterioridad, es un segmento que cuenta con cierta injerencia social relevante, tanto por su pasado, como por las labores que aún ejecutan desde las esferas políticas, laborales y dentro de los hogares.

¿Cómo beneficia la identidad a las personas de la tercera edad? Se realiza una actividad de reconocimiento social y no únicamente se las clasifica como un grupo vulnerable, ni como un segmento excluible; por el contrario, se bosqueja como una agrupación latente y predominante dentro del día a día que se exhibe en la cotidianidad y que es visible incluso dentro de los círculos cercanos a los sujetos, como el caso de la familia. Cabe destacar que la identidad es el derecho de ser diverso que:

... se desarrolla dentro de las pautas culturales e históricas, tradicionales o no, dentro de dinámicas de conflicto, con un periodo evolutivo propio $\mathrm{y}$ con un pasado y un futuro, con un conjunto de significaciones y representaciones que son relativamente permanentes. (Rojas, 2004, p. 490)

Para el caso de las personas de la tercera edad, su pasado representa el paso del tiempo que ha permitido el desarrollo y desenvolvimiento del presente, $y$ ha sido el primer armazón del futuro, es decir, es un referente de la experiencia previa que ha forjado la razón y motivación de las esferas sociales que nos rodean en la actualidad, de ahí que esto sea una razón para protegerles.

Desde los aspectos de los derechos humanos, la identidad es una garantía de permanencia y de resguardo del pasado, pues las vivencias $y$ experiencias de las personas adultas mayores tienden a ser el marco histórico por el cual se ha forjado todo lo que se conoce en la actualidad; emanciparles de los ámbitos sociales y humanos es la negación futura de la póstuma condición geriátrica de la juventud, es cuestión de tiempo llegar a la tercera edad, en ese entonces el presente será el pasado y de nuevo habrá que recurrir a la experiencia previa que permite la inclusión y garantizar la participación (en la vida cultural) de las personas de la tercera edad como sujetos sociales activos y latentes mediante las garantías humanas.

También la identidad funciona como un recordatorio sobre el deterioro del cuerpo humano, físico y mental, que en un futuro requerirá de ayuda por medio de instituciones que garanticen y salvaguarden los derechos humanos que permitan del desarrollo pleno e íntegro de cada ser a lo largo de su vida. Destaca también que el derecho a la identidad desemboca en los ámbitos de salud (atributos de las personas de la tercera edad), que pueden 
fungir como medida preventiva de enfermedades y para la realización de otros programas sociales que puedan erradicar o apaciguar los males que afecten a los sujetos.

Dicho sea de paso, las condiciones físicas y de salud repercuten en los ámbitos sociales y productivos de las ciudadanía, truncan y detienen el desarrollo óptimo de las labores realizadas para el mejoramiento del entorno en el que se vive, de ahí la importancia de la identidad o, en este caso, geroidentidad social ${ }^{13}$, pues es una aproximación a las necesidades y carencias que emanan de ciertos males sociales, que pueden ser erradicados desde los principios morales, que se fortalecen mediante las instituciones y que se heredan mediante la experiencia.

Una propuesta final es integrar los estudios sobre los derechos humanos de las personas de la tercera edad desde el enfoque de la identidad, pues se puede ampliar el espectro de necesidades, responsabilidades y garantías de dicho segmento poblacional, así mismo explicitar mucho más la importancia de la identidad dentro de las garantías humanas como el derecho a ser diverso o diversa y tener reconocimiento en las instancias sociales, jurídicas y humanas.

13 Se utiliza el término geroidentidad debido a una mezcla entre la etimología griega gero, que significa "anciano" (Pozzobon, 2011) con la palabra identidad para independizarle de otro tipo como la de carácter juvenil o psicológica.

\section{Conclusiones}

Pese a que dentro de las formas sociales en las que se conduce el ser humano exista una prominente actividad de clasificación que desemboca en etiquetas que tienden a estigmatizar a ciertos segmentos dentro del entorno actual, siempre habrá forma de concientizar a la sociedad sobre la independencia de un concepto instituido y sobre la vida de un segmento poblacional; esto no es más que un ejercicio de introspección y observación a los sitios que se concurren, a los lugares que se habitan, la gente con la que se lidia; todo ello puede generar un cambio en los niveles cognoscitivos y de acción, la tercera edad es un hecho que sucederá en algún momento para cada persona y la única responsabilidad que se tiene hasta llegar a ese punto es ser tolerante, comportarse con equidad y, por lo tanto, con empatía.

Dicho lo anterior, es menester que la sociedad priorice ciertos asuntos desde las diferentes perspectivas con las que se cuenta (ciudadanía, servicios públicos, dirigencias políticas, medios de comunicación, como madre, padre o familiar), puesto que el constante paso del tiempo tiende a olvidar sucesos que debieron ser tratados con mayor delicadeza, asuntos que van desde la inclusión familiar que desembocaron en el abandono, los tratos negligentes por considerarse supremo por ser 
joven, eso que gradualmente se irá deteriorando y que conllevará a todos los seres sociales a la tercera edad y que, de manera inevitable, habrá que encarar.

Las formas de gobierno, tanto estatales como federales han buscado siempre otorgar un beneficio universal a todos los niveles y esferas que componen el complejo sistema social que se vive y que se presta para un juego jerárquico, donde las autoridades cuentan con los medios para hacer llevadera la convivencia entre diferentes y diversos; pero siguen existiendo vacíos que no son satisfechos por las variadas necesidades que aquejan a la ciudadanía, como un reconocimiento social, que pudiera ser algo irrelevante e inclusive narcisista, pero que con la ejecución correcta brindaría cierto grado de visibilidad y evitaría encapsular a un segmento social en columnas de periódicos, debido a un acontecimiento desafortunado y que, posteriormente, será olvidado.

La identidad juega un papel crucial dentro de las formas de visibilidad social, ya que al conformarse a nivel individual, la persona adulta mayor se reconoce a sí misma a nivel colectivo, es decir, se hace una recopilación de lo que es el ser social, desde sus particularidades físicas, su proveniencia e historia y los sitios a los que acude junto con las personas que se sienten semejantes a él o a ella. Ahí es donde aquellos sujetos que no son, por ejemplo, parte de la tercera edad, asumen que esta población ocupa lugares dentro de los espacios que se transitan habitualmente y que tienen prácticas culturales diferentes a las de ellos; pero, no por eso, abandonan su condición ciudadana, de ser humano, hombre o mujer.

Esa medida diferenciadora permite que exista una imagen mental ${ }^{14}$ que será vinculada a un concepto previo: adulto mayor, grupo vulnerable, abuelo, anciana u algún otro eufemismo. Esto permite crear un patrón de reconocimiento, en donde se enuncian características físicas y una especulación de habilidades, pero jamás su historia, o en su caso, narrativa biográfica (Giménez, 2009), la cual se reconoce únicamente mediante la interacción; ya con el resguardo en la mente, se legitima la existencia de ese segmento poblacional.

Por eso, la identidad debería ser un derecho humano mucho más explícito dentro de las normativas, pues permite entender las diversas necesidades que aquejan a los sectores poblacionales vulnerables y optimiza procesos que estén referidos a estos mismos, dado que, como se mencionó anteriormente, existe un deterioro de habilidades

14 Las imágenes mentales son creadas mediante el ejercicio de la percepción y la reconstrucción de objetos visibles desde una perspectiva abstracta, es una referencia subjetiva de la realidad que se construye en la mente (Villafañe, 2006), se almacenan en la memoria $y$, por tanto, se conforman como un patrón de reconocimiento y vinculador de conceptos.

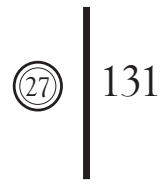


y capacidades (no universalmente) que dan pauta para la generación de violencia, discriminación y marginación, como consecuencia de la falta de entendimiento sobre la persona diferente.

No es raro que de la inconformidad surjan desacuerdos y con ellos movimientos sociales (no son las manifestaciones y bloqueos los problemas que resultan ser más relevantes para la ciudadanía, sino las acciones que se van a tomar para poder proponer soluciones a las problemáticas que aquejan a todos los que conforman las sociedades). Por supuesto que existen leyes y normativas o autoridades que se encarguen de lo anterior, pero, ¿qué hay de la acción humana?, ¿no existen dentro de las familias personas de la tercera edad?

Es labor y deber de cada ciudadano y ciudadana reconocerse y proyectarse en las personas de la tercera edad, pues permite entender las dificultades que rodean a dicho segmento, además de ser un ejercicio recopilatorio sobre lo que se debe hacer, lo que se hizo y lo que puede mejorar a futuro. La relación de la identidad con los derechos humanos parte del fundamento de la preocupación por el otro individuo, de resguardarlo y conocer a fondo las necesidades, pero también las aptitudes con las que cuenta para explotar todas sus garantías y que estas se vean reflejadas en bienestar, productividad y seguridad.

\section{Referencias}

Burgos, J. M. (2005). ¿Es la familia una institución natural? Cuadernos de Bioética, 16(3), 359-374.

Castoriadis, C. (1997). El imaginario social instituyente. Zona Erógena, 35, 1-9.

CNDH (Comisión Nacional de los Derechos Humanos). (2012). Derechos de los adultos mayores. Recuperado de http://www.cndh.org.mx/sites/ all/doc/cartillas/9 Cartilla Adultos Mayores.pdf

CONAPRED (Consejo Nacional para Prevenir la Discriminación). (2011). Encuesta nacional sobre discriminación en México / Enadis 2010. Recuperado de http://www.conapred.org.mx/ userfiles/files/Enadis-2010RG-Accss-002.pdf

CONAPO (Consejo Nacional de Población). (1999). Envejecimiento demográfico en México: Retos y perspectivas. Recuperado de http://www.gerontologia. org/portal/archivosUpload/ uploadManual/envejecimientomexico.pdf

De Lomnitz, L. A. (1998). Cómo sobreviven los marginados. Distrito Federal, México: Siglo Veintiuno Editores.

DOF (Diario Oficial de la Federación). (2015). Ley de los Derechos de las Personas Adultas Mayores. Recu- 
perado de http://www.sep.gob. $\mathrm{mx} /$ work/models/sep1/Resource/558c2c24-0b12-4676-ad908ab78086b184/ley derechos personas adultas mayores.pdf

DOF (Diario Oficial de la Federación). (2014). Acuerdo por el que se emiten las Reglas de Operación del Programa Pensión para Adultos Mayores, para el ejerciciofiscal2015. Recuperado de http://www.normateca. sedesol.gob.mx/work/models/ NORMATECA/Normateca/ Reglas_Operacion/2015/rop adultos_mayores.pdf

García, F. (Coord.) (2001). El siglo XX: Mirando hacia atrás para ver hacia delante. Madrid, España: Fundación para el Análisis y los Estudios Sociales.

García-Canclini, N.(2004).Diferentes, desiguales $y$ desconectados: Mapas de interculturalidad. Barcelona, España: Gedisa.

Galindo, I. (1981). Estudios de derecho civil. Distrito Federal, México: Universidad Nacional Autónoma de México.

Galtung, J. (2003). Violencia cultural. Bizkaia, España: Gernika Gogoratuz.

GEM (Gobierno del Estado de México). (30 de enero, 2014). Gaceta del Gobierno del Estado de México (19). Recuperado de http://legislacion.edomex. gob. $\mathrm{mx} /$ sites/legislacion. edomex.gob.mx/files/files/pdf/ gct/2014/ene306.PDF

Giménez, G. (2009). Identidades sociales. Distrito Federal, México: CONACULTA.

Halbwachs, M. (2004). La memoria colectiva. Zaragoza, España: Prensas Universitarias de Zaragoza.

Heller, H. (2000). Teoría del Estado. Distrito Federal, México: Fondo de Cultura Económica.

INAPAM (Instituto Nacional de las Personas Adultas Mayores). (2016). Beneficios y descuentos. En Beneficios tarjeta INA$P A M$. Distrito Federal, México: INAPAM. Recuperado de http://www.inapam.gob.mx/ en/INAPAM/Beneficios Tarjeta INAPAM

INEGI (Instituto Nacional de Estadística y Geografía). (2010). México en cifras. Información nacional, por entidad federativa y municipios. Aguascalientes, México: INEGI. Recuperado de http:// www3.inegi.org. $\mathrm{mx} /$ sistemas/ mexicocifras/default.aspx

La tercera edad. (Agosto 2011). La tercera edad. Características del adulto mayor Recuperado de http://www.horabuena.blogspot. $\mathrm{mx} / 2011 / 08 /$ la-tercera-edadcaracteristicas-del.html

Limón, M. R. (1992). Características psico-sociales de la tercera

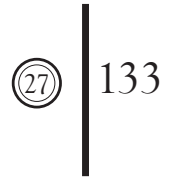


edad. Pedagogía Social: Revista Interuniversitaria, 7, 167-178.

New York-Presbyterian Hospital. (2005). Cambios de roles $y$ transiciones. Abuelos que crían a sus nietos (Folleto digital). New York, Estados Unidos: The University Hospitals of Columbia and Cornell, 1-9. Recuperado de http://www. cornellcares.org/pdf/handouts/ rct grandparents_sp.pdf

Pozzobon, A. (2011). Etimologia e abreviaturas de termos médicos. Um guía para estudantes, professores, autores e editores em medicina e ciencias relacionadas. Rio Grande do Sul, Brasil: Univates.

RAE (Real Academia de la Lengua Española). (2016). Vulnerable, En Diccionario de la lengua española (23a ed.). Madrid, España: RAE. Recuperado de http://dle.rae.es/?id=c5dW2by

Rodríguez, K. D. (2011). Vejez y envejecimiento. Documentos de investigación, 12, 1-42.

Rojas, M. (2004). Identidad y cultura. Educere, 8(27), 489-496.
SEDESOL (Secretaría de Desarrollo Social). (2015). Pensión para adultos mayores, en programas sociales. Distrito Federal, México: SEDESOL. Recuperado de http:// www.gob.mx/sedesol/acciones-y-programas/pesion-para-adultos-mayores?idiom $=\mathrm{es}$

SEDESOL (Secretaría de Desarrollo Social). (2015). Vinculación productiva de las personas adultas mayores, en programas sociales. Distrito Federal, México: SEDESOL. Recuperado de http://www.gob.mx/sedesol/ acciones-y-programas/vinculacion-productiva-de-las-personas-adultas-mayores?idiom $=\mathrm{es}$

Valadés, D., Gutiérrez, R. (Coords.) (2001). Derechos Humanos. MemoriadelIVCongresoNacional de Derecho Constitucional (Tomo III). Distrito Federal, México: Universidad Nacional Autónoma de México.

Villafañe, J. (2006). Introducción a la teoría de la imagen. Madrid, España: Ediciones Pirámide.

Recibido: 9/2/2016• Aceptado: 15/9/2016 